\title{
FRAT-up, a Web-based Fall-Risk Assessment Tool for Elderly People Living in the Community
}

Luca Cattelani ${ }^{1}$, PhD; Pierpaolo Palumbo ${ }^{1}$, MSc; Luca Palmerini ${ }^{1}$, PhD; Stefania Bandinelli ${ }^{2}$, MD; Clemens Becker ${ }^{3}$, $\mathrm{MD}$; Federico Chesani ${ }^{4}, \mathrm{PhD}$; Lorenzo Chiari ${ }^{1}, \mathrm{PhD}$

\footnotetext{
${ }^{1}$ Department of Electrical, Electronic, and Information Engineering - DEI, University of Bologna, Bologna, Italy

${ }^{2}$ Geriatric Unit, Azienda Sanitaria Firenze, Firenze, Italy

${ }^{3}$ Robert-Bosch-Krankenhaus Geriatric Rehabilitation Clinic, Stuttgart, Germany

${ }^{4}$ Department of Computer Science and Engineering - DISI, University of Bologna, Bologna, Italy
}

\section{Corresponding Author:}

Federico Chesani, PhD

Department of Computer Science and Engineering - DISI

University of Bologna

Viale Risorgimento 2

Bologna, 40136

Italy

Phone: 390512093086

Fax: 390512093869

Email: federico.chesani@unibo.it

\section{Abstract}

Background: About $30 \%$ of people over 65 are subject to at least one unintentional fall a year. Fall prevention protocols and interventions can decrease the number of falls. To be effective, a prevention strategy requires a prior step to evaluate the fall risk of the subjects. Despite extensive research, existing assessment tools for fall risk have been insufficient for predicting falls.

Objective: The goal of this study is to present a novel web-based fall-risk assessment tool (FRAT-up) and to evaluate its accuracy in predicting falls, within a context of community-dwelling persons aged 65 and up.

Methods: FRAT-up is based on the assumption that a subject's fall risk is given by the contribution of their exposure to each of the known fall-risk factors. Many scientific studies have investigated the relationship between falls and risk factors. The majority of these studies adopted statistical approaches, usually providing quantitative information such as odds ratios. FRAT-up exploits these numerical results to compute how each single factor contributes to the overall fall risk. FRAT-up is based on a formal ontology that enlists a number of known risk factors, together with quantitative findings in terms of odds ratios. From such information, an automatic algorithm generates a rule-based probabilistic logic program, that is, a set of rules for each risk factor. The rule-based program takes the health profile of the subject (in terms of exposure to the risk factors) and computes the fall risk. A Web-based interface allows users to input health profiles and to visualize the risk assessment for the given subject. FRAT-up has been evaluated on the InCHIANTI Study dataset, a representative population-based study of older persons living in the Chianti area (Tuscany, Italy). We compared reported falls with predicted ones and computed performance indicators.

Results: The obtained area under curve of the receiver operating characteristic was 0.642 (95\% CI 0.614-0.669), while the Brier score was 0.174 . The Hosmer-Lemeshow test indicated statistical significance of miscalibration.

Conclusions: FRAT-up is a web-based tool for evaluating the fall risk of people aged 65 or up living in the community. Validation results of fall risks computed by FRAT-up show that its performance is comparable to externally validated state-of-the-art tools. A prototype is freely available through a web-based interface.

Trial Registration: ClinicalTrials.gov NCT01331512 (The InChianti Follow-Up Study); http://clinicaltrials.gov/show/NCT01331512 (Archived by WebCite at http://www.webcitation.org/6UDrrRuaR).

(J Med Internet Res 2015;17(2):e41) doi: 10.2196/jmir.4064

\section{KEYWORDS}

accidental falls; odds ratio; risk assessment; risk factors; ROC curve; aged 


\section{Introduction}

\section{Background}

About $30 \%$ of community-dwelling people aged 65 or more experience at least one unintentional fall a year [1], and the annual fall rate in this cohort is about 0.65 falls per person [2]. Falls can result in injuries and are a leading cause of activity restriction, hospitalization, and disability [3,4]. Falling is the tenth leading cause of global years lived with disability (YLD). Worldwide, it accounts for about 20 million YLD [5] and a total of 35 million disability-adjusted life years [6]. Its burden is even more pronounced in countries with an older population; in Italy it is estimated to be the third leading cause of YLD [7].

Many preventive strategies have been proposed, and some of them have been shown to be effective [8-10]. Their implementation, however, has been slow and the coverage in Europe is insufficient [11-13]. The individual and societal costs of these interventions are often among the factors that hinder their implementation. In order to make use of available resources and intervene only with subjects at increased risk, medical associations and national health authorities recommend the adoption of fall-risk assessment tools [14-17].

\section{Existing Tools}

Reviews of fall-risk assessment tools and their accuracy are available in the literature [18-23]. Among the most used and validated tools are the Timed Up and Go Test (TUG), the Performance Oriented Mobility Assessment (POMA), and the Physiological Profile Assessment (PPA) [24]. Despite extensive research, existing assessment tools for fall risk have been insufficient for predicting falls [23,25-28].

\section{Existing Knowledge and Ontologies}

An impressive number of scientific publications have identified statistical correlation between the exposure to risk factors and the risk of falling, in terms of odds ratios. Moreover, several reviews and meta-analyses are available, thus providing a solid scientific base about fall-risk factors [29-35].

In our Fall-Risk Assessment Tool (FRAT-up), we faced the issue of representing the information available from scientific literature in a structured manner. In computer science, an ontology is a formal, explicit specification of a shared conceptualization [36]; ontologies are widely used in artificial intelligence, the semantic Web, and biomedical informatics as a form of knowledge representation. Formal approaches, like ontologies and the semantic Web, are important instruments also in epidemiology research [37].

\section{Aims of the Study}

The goal of FRAT-up is to provide a tool for the fall-risk assessment of subjects aged 65 or up and living in a community dwelling. The tool is mainly intended for two different health professional roles: (1) general practitioners (GPs) delivering primary care provisions, with no specific knowledge about falls, who need an assessment tool for evaluating subjects' fall risk and possible early interventions, and (2) professionals involved in fall prevention and treatment, who need a tool for constantly assessing the fall risk in a reliable and quantitative manner. We identified the following requirements: (1) the assessment tool should identify people at high risk of falling, (2) the tool should exploit existing knowledge about fall-risk factors, (3) the tool should be sufficiently flexible to allow the use of different clinical tests for the estimate of each risk factor, and (4) the assessment tool should be robust with respect to the unavailability of complete information about the subject.

FRAT-up has been developed within the FARSEEING Project [38], and it aims to meet all the requirements listed above.

\section{Methods}

\section{Overview}

The FRAT-up fundamental hypothesis is to consider the fall risk as being directly related to the subject's exposure to known risk factors. Thus, the starting point is the scientific literature that lists risk factors, together with quantitative information on their association with falls (usually in terms of odds ratios). However, such literature does not provide any structured definition of risk factors and related information. Hence, the first building block of the FRAT-up approach consists of a formal ontology listing risk factors and related data.

Once quantitative information is available through the FRAT-up risk factor ontology, we need to decide how (the exposure to) each risk factor contributes to the overall risk. Our approach is based on probabilities, while epidemiological studies on risk factors usually provide information in terms of odds ratios. Hence, the second building block is a mathematical transformation from odds ratios to probabilities under a few assumptions, as explained further in this section.

The third building block of FRAT-up is a Logic Programming with Annotated Disjunctions (LPAD) program that allows representation of the contribution of each risk factor in terms of probabilistic rules and probabilistic reasoning.

\section{A Formal Ontology for Fall-Risk Factors}

In FRAT-up, a fall-risk factor ontology has been defined, taking into account several domains. For example, the classification of risk factors by reversibility (surely reversible, subject specific reversible, or irreversible) and setting (community dwelling, acute care, etc) is shown in Figure 1. 
Figure 1. Classification of risk factors by kind, reversibility, and setting. While the InCHIANTI dataset is about community dwellings, the ontology covers other settings, too.

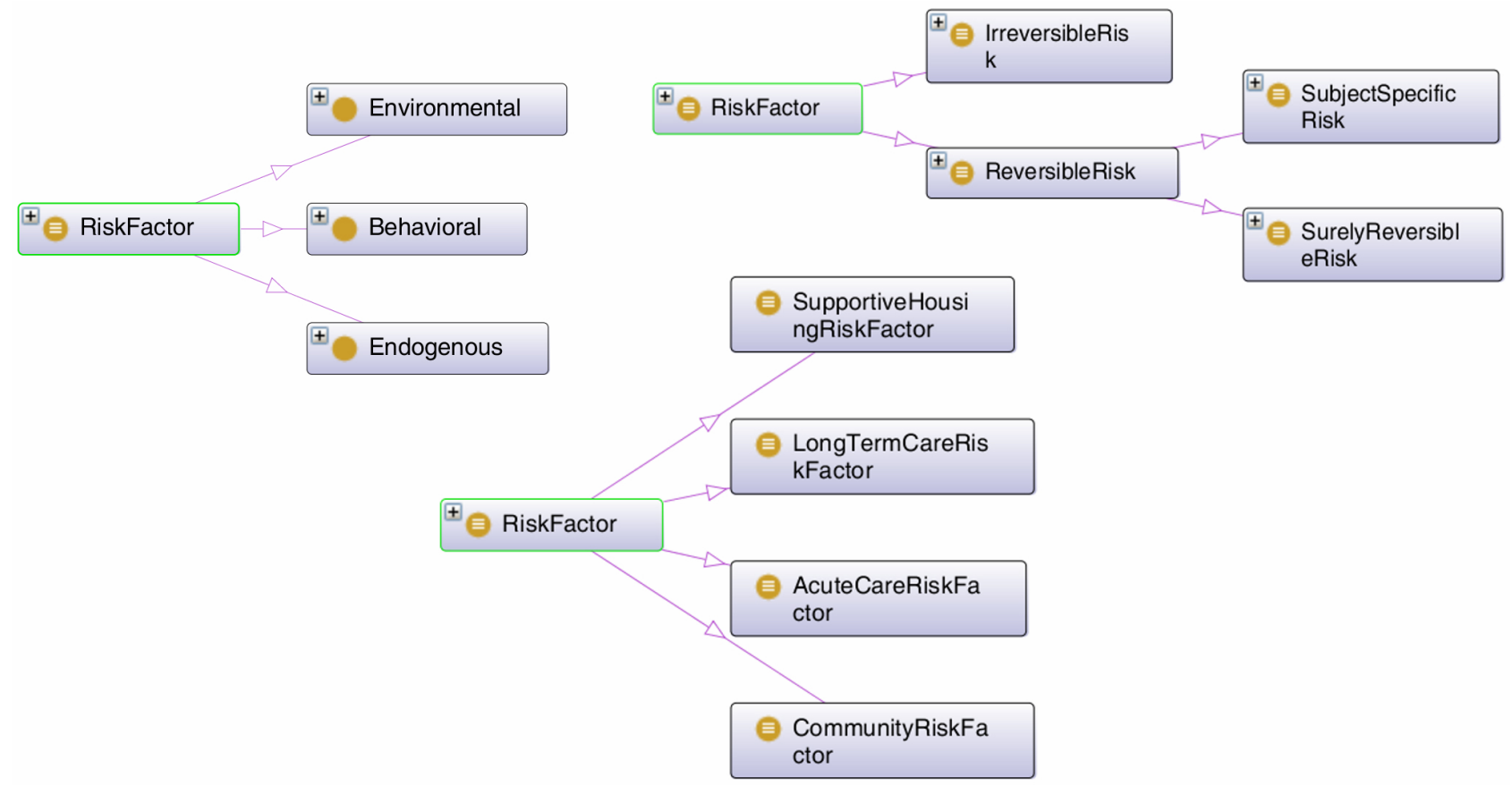

Within the ontology, risk factors are distinguished as dichotomous, scalar, and synergy factors. Dichotomous risk factors indicate whether a risky condition is present or not, without taking into account its severity. Scalar risk factors also indicate the magnitude of the subject's exposure to the risky condition. Since synergism between risk factors is well known [39], synergy factors make it explicit if two or more risk factors, due to their simultaneous presence, determine a higher risk than if present alone.

The set of risk factors we include in the ontology comes from a well-established meta-analysis on known risk factors for falls in community-dwelling older people by Deandrea et al [29] (see Table 1).

The ontology also includes the odds ratio for each risk factor, taken from Deandrea et al [29]. Moreover, we introduced in the ontology a clear distinction between a risk factor and the corresponding estimators. An estimator is a method to assess the presence and, when necessary, the severity of a risk factor (possibly in combination with other estimators).

Additional data contained in the ontology are the risk factors' prevalence and procedures to map estimators into factors. Complete information, including sources for quantitative data, is reported in Multimedia Appendix 1. 
Table 1. Risk factor names and types.

\begin{tabular}{|c|c|}
\hline Name & Type \\
\hline age & scalar \\
\hline cognition impairment & dichotomous \\
\hline depression & dichotomous \\
\hline diabetes & dichotomous \\
\hline comorbidity & synergy \\
\hline dizziness and vertigo & dichotomous \\
\hline fear of falling & dichotomous \\
\hline female sex & dichotomous \\
\hline gait problems & dichotomous \\
\hline hearing impairment & dichotomous \\
\hline history of falls & dichotomous \\
\hline history of stroke & dichotomous \\
\hline instrumental disability & dichotomous \\
\hline living alone & dichotomous \\
\hline number of medications & scalar \\
\hline pain & dichotomous \\
\hline parkinson & dichotomous \\
\hline physical activity limitation & dichotomous \\
\hline physical disability & dichotomous \\
\hline poor self-perceived health status & dichotomous \\
\hline rheumatic disease & dichotomous \\
\hline urinary incontinence & dichotomous \\
\hline use of antiepileptics & dichotomous \\
\hline use of antihypertensives & dichotomous \\
\hline use of sedatives & dichotomous \\
\hline vision impairment & dichotomous \\
\hline walking aid use & dichotomous \\
\hline
\end{tabular}

\section{From Odds Ratios to Probabilities}

\section{Overview}

The FRAT-up risk-assessment algorithm is based on probability contributions from single risk factors. In the following, we show how we extract probabilities from odds ratios by means of a few mathematical steps.

Initially, we assume that each risk factor is dichotomous; we explain this further in the section on how to generalize to cases with scalar and synergy risk factors. Let $E_{0}, E_{1}, \ldots, E_{n}$ be $n+1$ dichotomous random variables with values in $\{0 ; 1\}$, and $E=\left(E_{0}\right.$, $\left.E_{1}, \ldots, E_{n}\right)$. We say that the $i^{t h}$ risk factor is present if $E_{i}=1$. Let $d_{0}, d_{1}, \ldots, d_{n}$ be $n+1$ events. We assume the following conditional independence relations:

Equation $1: d_{i} \mid E_{i} \perp d_{j}, E_{j} \forall j \neq i$

We call $d_{i}$ a fall event specific to risk factor $E_{i}$. Assumptions from Equation 1 can be phrased saying that risk factor-specific falls are mutually independent conditional on their associated risk factor. We define the event $d$ as the union of the factor-specific events, $d_{i}$ 's (Figure 2). That is, $d$ is verified if at least one of the $d_{i}$ 's is verified. This is an assumption of causal independence where the "causes", $E_{0}, E_{1}, \ldots, E_{n}$, contribute independently to the probability of the effect $d$; for a complete formal definition see [40]. In our case study, $d$ is the presence of at least one fall event during a given time span (if there is no fall, it is not verified), while $E$ is an observation of the risk factor exposures of a subject before the time span.

The conditional probability of $d$ given $E$ can then be calculated as in Figure 3, by De Morgan laws and assumptions in Equation 1. This function models the probability of an event given a set of possible causes and is known as noisy-OR gate [41] (in this case OR refers to the logical operator). We make the assumption in Figure 4. $C_{i}$ is a quantity yet to determine. $C_{i}$ is the contribution to the probability of the effect $d$ given by the exposure to the risk factor $E_{i}$. A method to assign values to the 
contributions $C_{i}$ is introduced in the following. Using the equation in Figure 4, the equation in Figure 3 becomes the one depicted in Figure 5. Since we want to model a minimum probability of the adverse event that is applied even in the absence of any observation-specific exposures, we assign $P\left(E_{0}=1\right)=1 . C_{0}$ is the risk that is present in this case. To assign values to the contributions of the exposures, we start from the OR. The OR relative to risk factor $E_{i}$, with $i=1, \ldots, n$, is defined as in Figure 6. Note that the condition $E_{0}=1$ is always true and is highlighted above just for convenience.

There is no single way of translating odds ratios to probabilities, since an exact function would require more information than what is conveyed by the odds ratios alone, so some assumptions are needed. We present a possible set of assumptions that leads to a univocal way of computing exposure contributions.

Figure 2. Definition of fall event.

$$
d:=\bigcup_{i=0}^{n} d_{i}
$$

Figure 3. Probability to fall from risk factor specific probabilities.

$$
\begin{aligned}
P(d \mid E)= & P\left(\bigcup_{i=0}^{n} d_{i} \mid E\right)=1-P\left(\overline{\bigcup_{i=0}^{n} d_{i}} \mid E\right)=\quad(a) \\
& =1-P\left(\bigcap_{i=0}^{n} \bar{d}_{i} \mid E\right)= \\
& =1-\prod_{i=0}^{n} P\left(\bar{d}_{i} \mid E_{i}\right)= \\
& =1-\prod_{i=0}^{n}\left[1-P\left(d_{i} \mid E_{i}\right)\right]
\end{aligned}
$$

\section{Assumption (a)}

We assume that $O R_{i}$ may be approximated as in Figure 7. Informally, Assumption (a) states that the odds ratio computed on the whole population is similar to the odds ratio computed restricting the population to subjects having at most one exposure. This assumption is obviously true in models where each subject has at most one exposure; otherwise there is a difference in the two values. This has not been quantified yet; the quality of the approximation will be experimentally compared with other methods as a future development.

Given the assumptions in Equation 1 and Figure 4, the derivation depicted in Figure 8 follows. Substituting the equation in Figure 8 in the equation in Figure 7 and solving for $C_{i}$, we finally get the equation depicted in Figure 9. We substitute it in the equation in Figure 5, with a result that is depicted in Figure 10.

Figure 4. Probability of factor specific fall event given exposure.

$$
P\left(d_{i} \mid E_{i}\right)=\left\{\begin{array}{ccc}
0 & \text { if } & E_{i}=0 \\
C_{i} & \text { if } & E_{i}=1
\end{array}\right.
$$

Figure 5. Probability to fall given exposures and contributions.

$$
P(d \mid E)=1-\prod_{i=0}^{n}\left(1-E_{i} C_{i}\right)
$$

Figure 6. Odds ratio definition.

$$
O R_{i}:=\frac{P\left(d \mid E_{0}=1, E_{i}=1\right)}{1-P\left(d \mid E_{0}=1, E_{i}=1\right)} \frac{1-P\left(d \mid E_{0}=1, E_{i}=0\right)}{P\left(d \mid E_{0}=1, E_{i}=0\right)}
$$

Figure 7. Approximated odds ratio.

$$
O R_{i}=\frac{P\left(d \mid E_{0}=1, E_{i}=1, E_{j \neq 0, i}=0\right)}{1-P\left(d \mid E_{0}=1, E_{i}=1, E_{j \neq 0, i}=0\right)} \frac{1-C_{0}}{C_{0}}
$$


Figure 8. Probability to fall with exposure to exactly 1 risk factor.

$$
\begin{gathered}
P\left(d \mid E_{0}=1, E_{i}=1, E_{j \neq 0, i}=0\right)= \\
=P\left(d_{0} \vee d_{i} \mid E_{0}=1, E_{i}=1, E_{j \neq 0, i}=0\right)= \\
P\left(d_{0} \mid E_{0}=1\right)+P\left(d_{i} \mid E_{i}=1\right)-P\left(d_{0} \mid E_{0}=1\right) P\left(d_{i} \mid E_{i}=1\right)= \\
C_{0}+C_{i}-C_{0} C_{i}
\end{gathered}
$$

Figure 9. Contribution to fall probability from exposure to a single risk factor given odds ratio.

$$
C_{i}=C_{0} \frac{O R_{i}-1}{1-C_{0}+C_{0} O R_{i}}
$$

Figure 10. Probability to fall from risk factor odds ratios.

$$
P(d \mid E)=1-\left(1-C_{0}\right) \prod_{i=1}^{n}\left(1-E_{i} C_{0} \frac{O R_{i}-1}{1-C_{0}+C_{0} O R_{i}}\right)
$$

\section{Assumption (b)}

We assume to know $C_{0}$, which was calculated by leaving it as a free parameter and then learning it with an equation-solving algorithm. In particular, we used the bisection method, imposing the reported number of total falls from [1].

This model requires that we know for every risk factor if it is present or not. In the following section, we present the way FRAT-up deals with missing values.

For a general reference on how to get relative risk from odds ratio and the incidence of the outcome of interest in the unexposed group, see [42].

\section{LPAD Structure and Handling of Unknown Exposures, and Scalar and Synergy Risk Factors}

LPADs are logic programs [43] where the head of a clause is a disjunction of annotated atoms. The clauses are of the form:

$h_{1}: p_{1} \mathrm{\vee} \ldots \vee h_{n}: p_{n} \leftarrow b_{1} \wedge \ldots \wedge b_{m} \wedge c_{1} \wedge \ldots \wedge c_{l}$

where $h_{1}, \ldots, \quad h_{n}$ are the atoms, and $p_{1}, \ldots, p_{n}$ are the probabilities related to each disjunct. Each atom $h_{i}$ has probability $p_{i}$ if the body is true, and the atom does not appear in the head of any other clause. When it does, the intended semantics are the distribution semantics as in [44], with the bodies contributing independently to the probability of the atom [40]. The probabilities $p_{1}, \ldots, p_{n}$ should sum up to 1 , with an implicit "null" atom when the explicit probabilities sum up to less than 1.

Roughly speaking, for each clause containing a disjunction in its head, different instances are generated, each containing the clause with exactly one disjunct. The probability of a query would be given by the sum of all the probabilities of the instances whose models contain it.

We adopt the syntax of the cplint [45] implementation. Note that the disjunction in the head of clauses is indicated with the symbol ";", while the conjunction is indicated as usual in Prolog with ",". The equation in Figure 5 can be easily implemented with LPAD rules (Code 1 LPAD template with computed fall probability contributions):

$$
\begin{aligned}
& \text { fall(X) : c0. } \\
& \text { fall }(X): c 1:-e 1(X) \\
& \text { fall(X) : c2 :- e2(X). }
\end{aligned}
$$

$\cdots$

Where $\mathrm{c} 0 \equiv C_{0}, \mathrm{c} 1 \equiv C_{1}, \mathrm{e} 1(\mathrm{X}) \equiv\left(E_{1}=1\right), \mathrm{c} 2 \equiv C_{2}, \mathrm{e} 2(\mathrm{X}) \equiv\left(E_{2}=1\right)$

The assessment tool should provide reliable information even when part of the subject's data is missing. Missing values may arise when a test has not been (or cannot be) performed or the involved clinical professional does not consider its outcome decisive and reliable. In these cases, we have used the prevalence of the risk factors extending Code 1 as follows:

fall $(\mathrm{X}): \mathrm{c} 0$.

e1(X) : p1 :- u1(X).

fall(X) : c1 :- e1(X).

$\mathrm{e} 2(\mathrm{X}): \mathrm{p} 2$ :- u2(X).

fall(X) : c2 :- e2(X).

$\cdots$

where $\mathrm{u} 1(\mathrm{X}), \mathrm{u} 2(\mathrm{X}) \ldots$ is true when the existence of the factor $1,2 \ldots$ for subject $X$ is not determined.

A scalar factor, with exposure levels from 0 (no exposure) to $m$ (maximum exposure), is implemented similarly to a set of $m$ dichotomous factors, one for each exposure level starting from level 1. The LPAD rule related to level $k$ fires if the scalar risk factor has a level of $k$ or higher.

Positive synergies (eg, comorbidities) between risk factors are well documented in the scientific literature. Since this would violate the causal independence assumption made before, we adjusted the model, following the Deandrea meta-analysis [29], introducing synergy factors. 
A synergy factor, representing the potential synergies between $S$ dichotomous risk factors, is implemented similarly to a scalar risk factor having a maximum possible level of $S-1$ where, having a number of exposures equal to $q$, with $0 \leq q \leq S$, the level is 0 if $q=0 \mathrm{v} q=1$ and is $q$ - 1 otherwise. So the risk starts increasing when there is a synergy between at least two factors.

\section{Automatic Generation of the LPAD}

The methodology that leads from risk factor odds ratio to LPAD rules is fully automatized. A working prototype has been produced and tested in the Java programming language (version
1.7); it may read risk factor odds ratios from an ontology or another source and outputs an LPAD program directly usable for risk assessment.

Synthetically (see Figure 11), risk factors data complete with odds ratio are read from an ontology or other data source; a data structure containing odds ratios is created and then transformed (by means of the equation in Figure 9) in another containing probability values. Finally LPAD rules are compiled: these rules are applied to a subject to give their probability of falling in a given time span.

Figure 11. Steps in generating the LPAD rules.

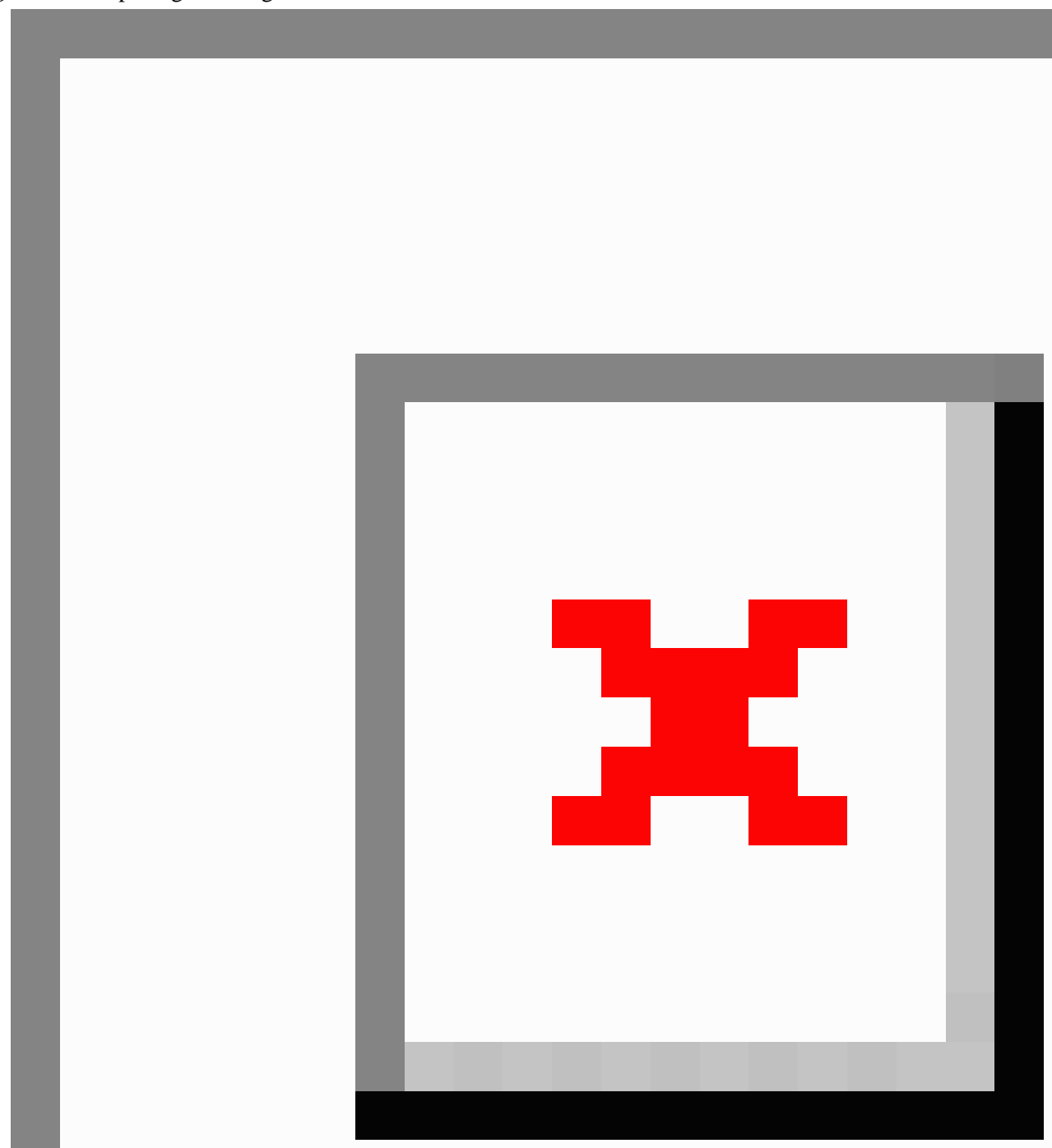




\section{Dataset and Validation Procedure}

FRAT-up discriminative performance and calibration have been tested on the InCHIANTI dataset (NCT01331512), where 1453 persons have been initially enrolled (1150 subjects aged 65 or more) and have undergone four consecutive visits globally covering a 9-year follow-up. It is a population-based epidemiologic study conducted in the Chianti region of Italy in two sites: Greve in Chianti (Area 1; 11,709 inhabitants; >65 years: 19.3\%) and Bagno a Ripoli (Village of Antella, Area 2, 4704 inhabitants; $>65$ years: $20.3 \%$ ). This study investigates age-related decline in mobility [46].

The InCHIANTI study started in September 1998 with the baseline assessment (first wave), which was completed in March 2000. Every 3 years, a follow-up assessment was performed. So, 3-year and 6-year follow-up assessments were performed respectively in 2001-2003 and 2004-2006 (second and third wave). A 9-year follow-up was then performed in 2007-2009 (fourth wave). The fifth wave is now ongoing.

At each wave, subjects were asked about the occurrence of any fall in the previous 12 months. In addition, clinical evaluation of the subjects was performed to collect information on fall-risk factors (other clinical variables were also collected, which are not of interest for this work [46]).

Our study used the information about risk factors from the first three waves, considering only subjects aged 65 or up. By doing so, we obtained 2319 samples from 977 subjects (every subject can have up to three samples).

At each wave, the risk factors of each subject were used prospectively to calculate their risk of falling at the subsequent wave (eg, the risk factors from the clinical evaluation at baseline were used to calculate the future risk of falling, which was compared with the recorded information on the occurrence of any falls in the 12 months before follow-up 1, and so on).

The estimators present in the InCHIANTI dataset and the algorithms to derive the risk factors from them are listed in Multimedia Appendix 1.

The discriminative ability and calibration of FRAT-up were validated by means of receiver operating characteristic (ROC) curve, area under the ROC curve (AUC), Brier score, and the Hosmer-Lemeshow test [47]. Since FRAT-up requires no training of the algorithm based on the available data, these metrics were computed by using all the available data as the test set.

\section{Results}

The ROC curve can be seen in Figure 12; the AUC value is 0.642 (95\% CI 0.614-0.669). The Hosmer-Lemeshow test produces a very low $P$ value $(<.001)$ indicating statistical significance of miscalibration. As shown by the calibration plot in Figure 13, this miscalibration is due to risk overestimation that is consistent over the risk strata. The Brier score is 0.174 . 
Figure 12. ROC curve obtained on the InCHIANTI dataset.

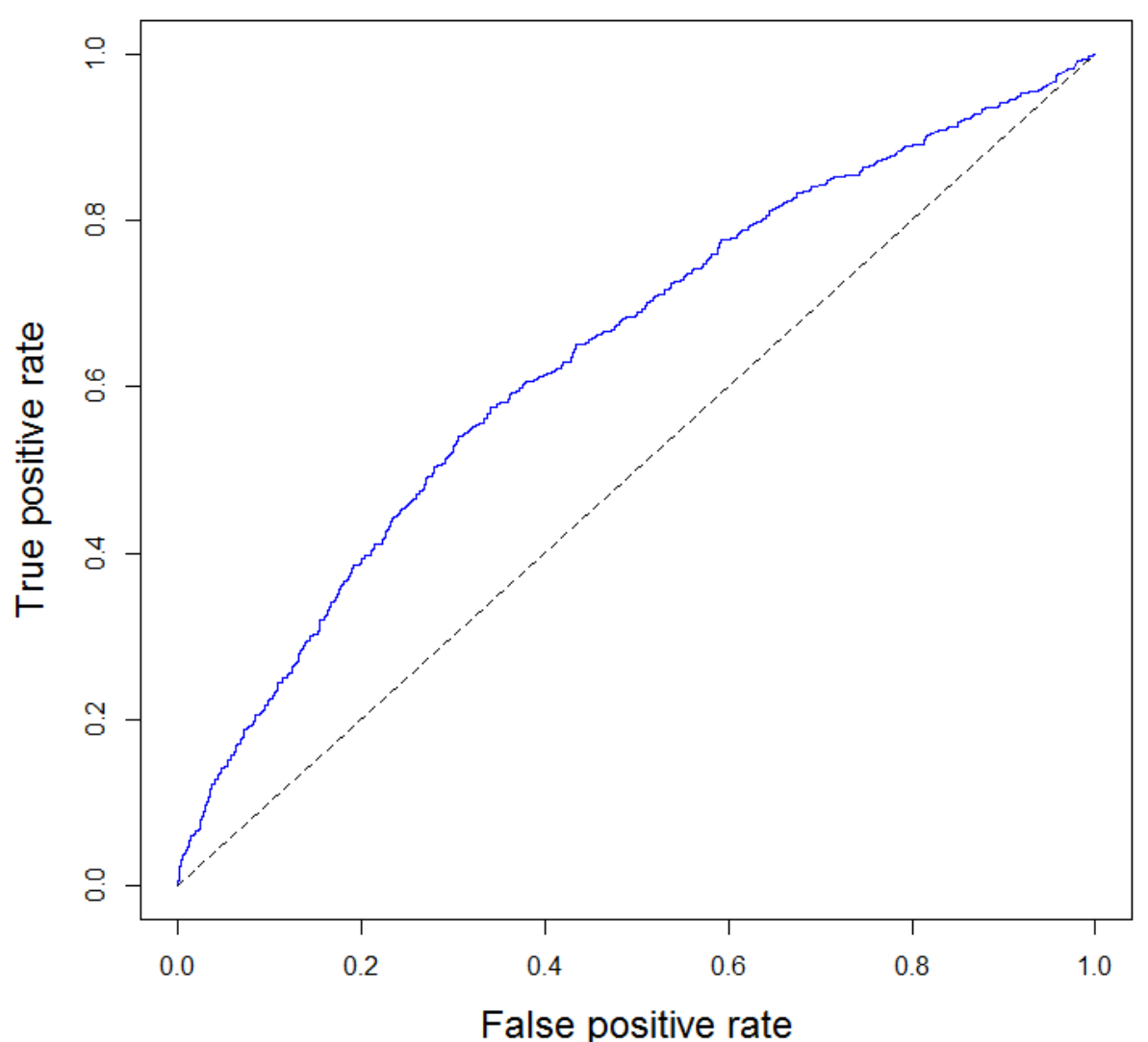


Figure 13. Calibration plot; sample $(\mathrm{N}=2319)$ used for validation where divided in 10 deciles, according to their predicted risk. For each decile, the mean predicted risk and the observed proportion of positive cases (proportion of fallers) are shown on the $\mathrm{X}$ and $\mathrm{Y}$ axes, respectively. Bars indicate $95 \%$ confidence intervals.

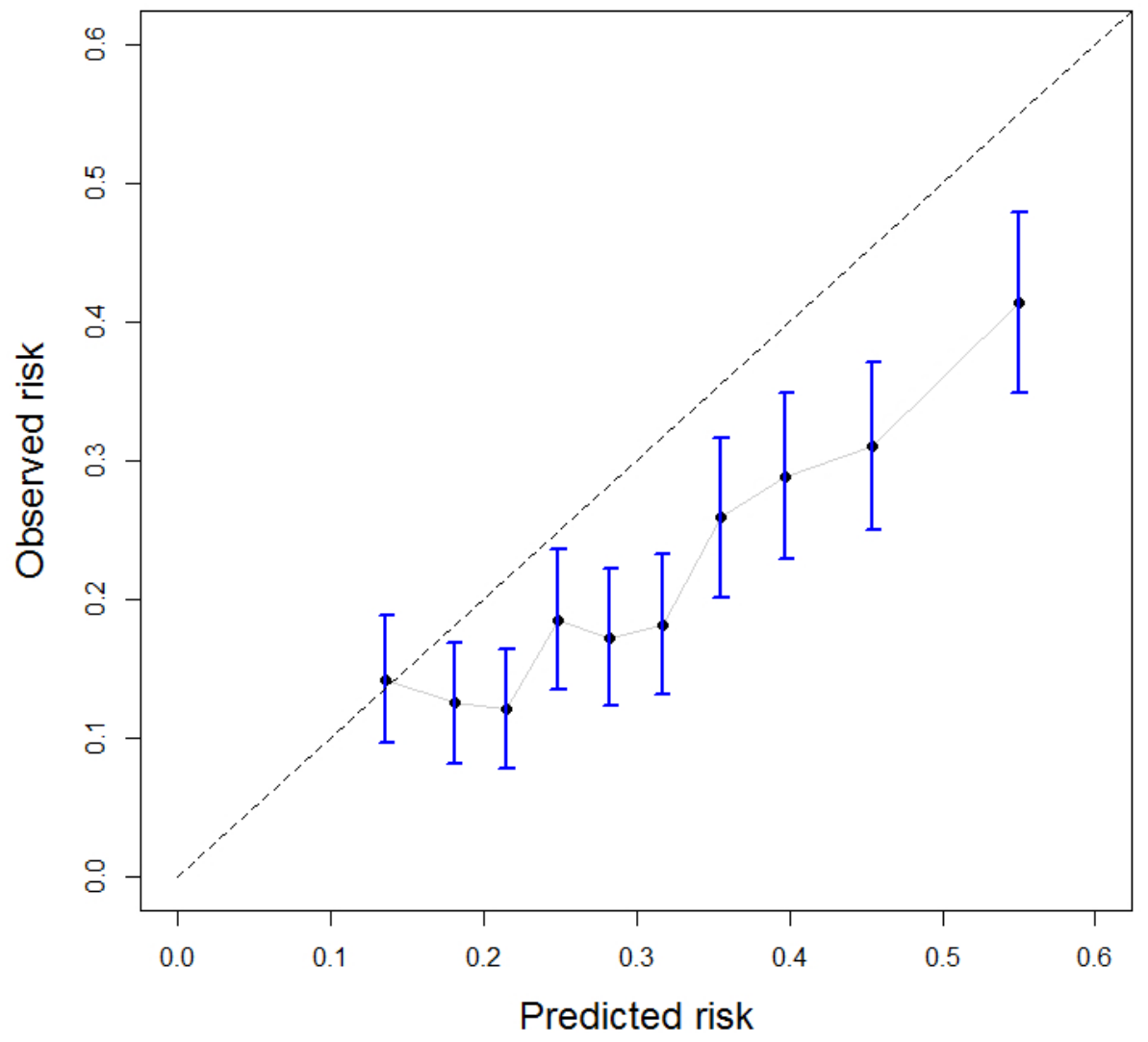

\section{Discussion}

\section{Principal Findings}

The ability to discriminate between subjects who fall and subjects who do not fall, as operationalized by the AUC (0.642), compares favorably with other commonly used screening tools: a recent meta-analysis has estimated that the AUC of the TUG is between 0.54 and 0.59 [28], while the POMA-balance (also known as Tinetti balance scale) has AUC around 0.56 [23]. Since at each wave of the study, each subject was asked whether they had fallen in the last 12 months and the waves were about 3 years apart, this means we evaluated a prediction for an event that materializes between about 24 and 36 months after the assessment of the risk factors. Had the information about falls been available for the year just after the assessment, the results would likely have been better. Additionally, it is worth noting that the InCHIANTI dataset was not specifically designed to investigate fall risk. Because of these limitations, validation on other datasets would be desirable.

FRAT-up overestimates the fall risk. Since this overestimation, as shown in Figure 13, is consistent across deciles, the miscalibration is of less concern. The main reason behind this overestimation could be that the incidence of falls from [1] ( $31 \%$ subjects fallen at least once in a year), which was used for calculating the term $C_{0}$, is higher than the observed incidence of falls in the InCHIANTI population (22\%). A possible way to reduce overestimation would be multiplying the output by a constant, but we did not exploit this kind of learning on the dataset.

FRAT-up does exploit existing knowledge as it was built only from information derived from the literature, which was systematized in a meta-analysis. By doing so, it avoids overfitting and overoptimism, problems well known to affect predictive models [48].

Although the validation on the InCHIANTI dataset is based on a specific set of estimators, the architecture allows for the use of different estimators. The results of the validation have been obtained from the InCHIANTI dataset, where the percentage of missing values ranges from $0 \%$ on some variables (eg, sex and age) to $17 \%$ on vision impairment.

The interactive prototype of the FRAT-up algorithm is freely available online [49]. Its interface is depicted in Figure 14. 
Figure 14. Screenshot of the Web-based interface.
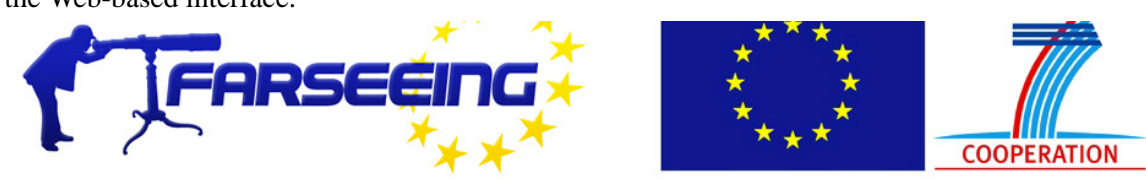

\section{FRAT-up \\ Fall Risk Assessment Tool}

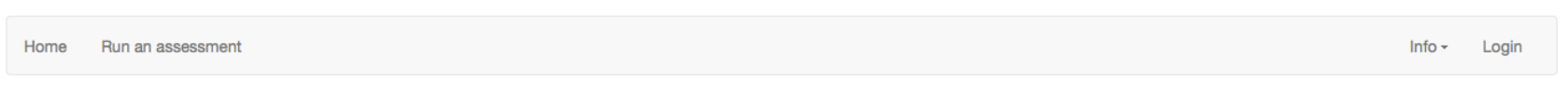

Current risk of the subject: Unknown

Health profile of the subject:

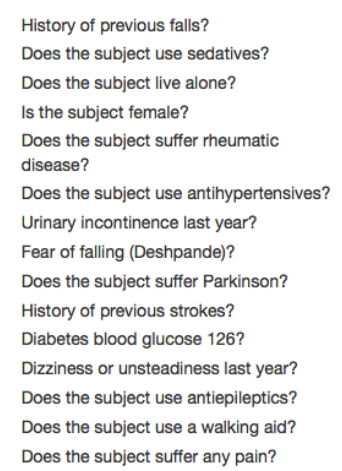

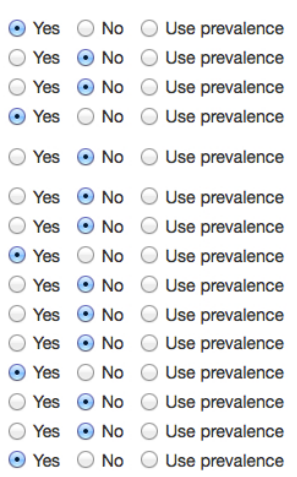

$\odot$ Yes $\bigcirc$ No $\bigcirc$ Use prevalence

\section{Limitations and Future Developments of FRAT-up}

FRAT-up is based on the simplifying assumption that the risk factors contribute independently to the probability of falling. Following Deandrea et al [29] and to partially relax this assumption, we introduced the synergy factors in the methods section. However, different approaches may be investigated.

Our method showed robustness with respect to the missing values present in the InCHIANTI dataset. However, the extent to which the presence of missing values impacts the performance should be further investigated. In the future, FRAT-up will be tested on other datasets of different populations, possibly with different estimators for the risk factors, and compared with alternative risk assessment tools. Additionally, considering confidence intervals of the odds ratios could allow us in the future to assess the uncertainty associated with the fall-risk estimation.

Risk factors not reported in the meta-review by Deandrea et al [29] (such as rare risk factors) are not considered. Also, other information sources like experts opinion and administrative/demographic data are currently ignored. Ongoing work is devoted to extend the risk factor ontology with this additional information. The evaluation of the tool should go beyond statistical assessment alone. Usability and usefulness, which are increasingly acknowledged as important in the literature of prognostic models [50], will hence be evaluated.

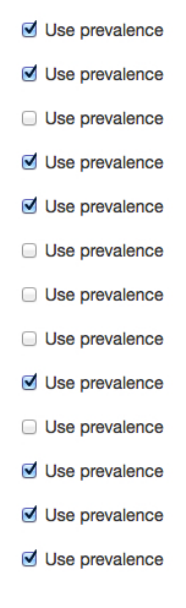

Within the framework of a fall-prevention strategy, information would be useful on the indication of the modifiable risk factors of a specific subject and their quantitative impact on their risk. Practically, we foresee integration of the tool within electronic medical records, tools of general practitioners, as well as its adoption in public health bodies for population-wide evaluation.

The versatility of the presented solution will allow combining clinical information (that was used in this study) with other sources of data such as ambient sensor information or wearable sensors recording unsupervised long-term physical activity and/or quantitatively evaluating supervised or unsupervised physical performance by instrumented motor assessment [51-55].

An interesting extension of FRAT-up would be to implement it as an app for "smart" devices such as smartphones. The tool might be fed with rich sensor-based information and could be extended to provide "real-time" risk evaluation based on the subject's current physical activity. Although from the technical viewpoint, such an extension would be easy and straightforward, using smartphone sensor data (in the fall-risk estimation) is still an open research issue.

Finally, since FRAT-up is based on a general methodology, it may be extended/applied in different ways, such as estimating fall risks in different settings (eg, acute care or nursing homes). Another extension would be to estimate outcomes other than falling, such as stroke risk, and more generally, estimate any risks directly related to the presence/absence of risk factors. 


\section{Acknowledgments}

The tool has been designed and implemented by LC, PP, LP, FC, and LC (Prof. Lorenzo Chiari) who coordinates the research group in the University of Bologna. SB and CB contributed to general discussion of the idea, the identification of the requirements, as well as the interpretation of the data and results. SB, as InCHIANTI principal investigator, has contributed the dataset.

The research leading to these results has received funding from the European Union Seventh Framework Programme (FP7/2007-2013) under grant agreement FARSEEING No. 288940.

The authors would like to thank Marco Colpo for his valuable help with the InCHIANTI dataset.

\section{Conflicts of Interest}

Clemens Becker has received consultation fees by Eli Lilly and Robert Bosch GmbH.

\section{Multimedia Appendix 1}

FRAT-up estimators, factors, and procedures to produce factor values starting from estimator values.

\section{[PDF File (Adobe PDF File), 153KB-Multimedia Appendix 1]}

\section{References}

1. World Health Organization Department of Ageing and Life Course. WHO Global Report on Falls Prevention in Older Age. Geneva, Switzerland: World Health Organization; 2008.

2. Rubenstein LZ. Falls in older people: epidemiology, risk factors and strategies for prevention. Age Ageing 2006 Sep;35 Suppl 2:ii37-ii41 [FREE Full text] [doi: 10.1093/ageing/afl084] [Medline: 16926202]

3. Gill TM, Allore HG, Holford TR, Guo Z. Hospitalization, restricted activity, and the development of disability among older persons. JAMA 2004 Nov 3;292(17):2115-2124. [doi: 10.1001/jama.292.17.2115] [Medline: 15523072]

4. Mathers LJ, Weiss HB. Incidence and characteristics of fall-related emergency department visits. Acad Emerg Med 1998 Nov;5(11):1064-1070. [Medline: 9835467]

5. Vos T, Flaxman AD, Naghavi M, Lozano R, Michaud C, Ezzati M, et al. Years lived with disability (YLDs) for 1160 sequelae of 289 diseases and injuries 1990-2010: a systematic analysis for the Global Burden of Disease Study 2010. Lancet 2012 Dec 15;380(9859):2163-2196. [doi: 10.1016/S0140-6736(12)61729-2] [Medline: 23245607]

6. Murray CJ, Vos T, Lozano R, Naghavi M, Flaxman AD, Michaud C, et al. Disability-adjusted life years (DALYs) for 291 diseases and injuries in 21 regions, 1990-2010: a systematic analysis for the Global Burden of Disease Study 2010. Lancet 2012 Dec 15;380(9859):2197-2223. [doi: 10.1016/S0140-6736(12)61689-4] [Medline: 23245608]

7. Global Burden of Disease Study 2010. URL: http://vizhub.healthdata.org/irank/arrow.php [accessed 2015-01-28] [WebCite Cache ID 6Vuqk38fI]

8. Kannus P, Sievänen H, Palvanen M, Järvinen T, Parkkari J. Prevention of falls and consequent injuries in elderly people. Lancet 2005 Nov 26;366(9500):1885-1893. [doi: 10.1016/S0140-6736(05)67604-0] [Medline: 16310556]

9. Gillespie LD, Robertson MC, Gillespie WJ, Sherrington C, Gates S, Clemson LM, et al. Interventions for preventing falls in older people living in the community. Cochrane Database Syst Rev 2012;9:CD007146. [doi:

10.1002/14651858.CD007146.pub3] [Medline: 22972103]

10. Cameron ID, Gillespie LD, Robertson MC, Murray GR, Hill KD, Cumming RG, et al. Interventions for preventing falls in older people in care facilities and hospitals. Cochrane Database Syst Rev 2012;12:CD005465. [doi: 10.1002/14651858.CD005465.pub3] [Medline: 23235623]

11. Falling standards, broken promises. In: Falling standards, broken promisesbased on findings from the national audit of falls and bone health in older people 2010. London: Royal College of Physicians; 2010.

12. Royal College of Physicians. Report of the 2011 inpatient falls pilot audit. London: RCP; 2012.

13. Center of Research Excellence in Patient Safety. An evaluation of the preventing falls and harm from falls in older people best practice guidelines for Australian hospitals. In: Australian Commission on Safety and Quality in Health Care. Australia: Australian Commission on Safety and Quality in Health Care; 2012.

14. Panel on Prevention of Falls in Older Persons, American Geriatrics Society and British Geriatrics Society. Summary of the Updated American Geriatrics Society/British Geriatrics Society clinical practice guideline for prevention of falls in older persons. J Am Geriatr Soc 2011 Jan;59(1):148-157. [doi: 10.1111/j.1532-5415.2010.03234.x] [Medline: 21226685]

15. Brigoni P, De Masi S, Di Franco M, Rinonapoli G, Romanini E, Sampaolo L. Sistema nazionale per le linee guida - Istituto superiore di sanità. Roma; 2007. Prevenzione delle cadute da incidente domestico negli anziani URL: http://www.snlg-iss.it/ lgn cadute da incidente domestico negli anziani [accessed 2015-01-28] [WebCite Cache ID 6VusbH4TC]

16. Barker W. Assessment and prevention of falls in older people. Nurs Older People 2014 Jul;26(6):18-24. [doi: 10.7748/nop.26.6.18.e586] [Medline: 24975078] 
17. Beauchet O, Dubost V, Revel Delhom C, Berrut G, Belmin J, French Society of Geriatrics and Gerontology. How to manage recurrent falls in clinical practice: guidelines of the French Society of Geriatrics and Gerontology. J Nutr Health Aging 2011 Jan;15(1):79-84. [Medline: 21267524]

18. Fabre JM, Ellis R, Kosma M, Wood RH. Falls risk factors and a compendium of falls risk screening instruments. J Geriatr Phys Ther 2010;33(4):184-197. [Medline: 21717922]

19. Scott V, Votova K, Scanlan A, Close J. Multifactorial and functional mobility assessment tools for fall risk among older adults in community, home-support, long-term and acute care settings. Age Ageing 2007 Mar;36(2):130-139 [FREE Full text] [doi: 10.1093/ageing/afl165] [Medline: 17293604]

20. Oliver D, Daly F, Martin FC, McMurdo ME. Risk factors and risk assessment tools for falls in hospital in-patients: a systematic review. Age Ageing 2004 Mar;33(2):122-130 [FREE Full text] [doi: 10.1093/ageing/afh017] [Medline: 14960426]

21. Perell KL, Nelson A, Goldman RL, Luther SL, Prieto-Lewis N, Rubenstein LZ. Fall risk assessment measures: an analytic review. J Gerontol A Biol Sci Med Sci 2001 Dec;56(12):M761-M766. [Medline: 11723150]

22. Close JC, Lord SR. Fall assessment in older people. BMJ 2011;343:d5153. [Medline: 21917828]

23. Gates S, Smith LA, Fisher JD, Lamb SE. Systematic review of accuracy of screening instruments for predicting fall risk among independently living older adults. J Rehabil Res Dev 2008;45(8):1105-1116 [FREE Full text] [Medline: 19235113]

24. Lord SR, Menz HB, Tiedemann A. A physiological profile approach to falls risk assessment and prevention. Phys Ther 2003 Mar;83(3):237-252 [FREE Full text] [Medline: 12620088]

25. Oliver D. Assessing the risk of falls in hospitals: time for a rethink? Can J Nurs Res 2006 Jun;38(2):89-94; discussion 95. [Medline: 16871852]

26. Morse JM. The safety of safety research: the case of patient fall research. In: CJNR (Canadian J Nurs Res McGill School of Nursing). Canada: McGill School of Nursing; 2006:74-88.

27. Oliver D. Falls risk-prediction tools for hospital inpatients. Time to put them to bed? Age Ageing 2008 May;37(3):248-250 [FREE Full text] [doi: 10.1093/ageing/afn088] [Medline: 18456789]

28. Barry E, Galvin R, Keogh C, Horgan F, Fahey T. Is the Timed Up and Go test a useful predictor of risk of falls in community dwelling older adults: a systematic review and meta-analysis. BMC Geriatr 2014;14:14 [FREE Full text] [doi: 10.1186/1471-2318-14-14] [Medline: 24484314]

29. Deandrea S, Lucenteforte E, Bravi F, Foschi R, La Vecchia C, Negri E. Risk factors for falls in community-dwelling older people: a systematic review and meta-analysis. Epidemiology 2010 Sep;21(5):658-668. [doi:

10.1097/EDE.0b013e3181e89905] [Medline: 20585256]

30. Lord SR, Sherrington C, Menz HB. Falls in Older People: Risk Factors and Strategies for Prevention. Cambridge: Cambridge University Press; 2001.

31. Leipzig RM, Cumming RG, Tinetti ME. Drugs and falls in older people: a systematic review and meta-analysis: II. Cardiac and analgesic drugs. J Am Geriatr Soc 1999 Jan;47(1):40-50. [Medline: 9920228]

32. Leipzig RM, Cumming RG, Tinetti ME. Drugs and falls in older people: a systematic review and meta-analysis: I. Psychotropic drugs. J Am Geriatr Soc 1999 Jan;47(1):30-39. [Medline: 9920227]

33. Moreland JD, Richardson JA, Goldsmith CH, Clase CM. Muscle weakness and falls in older adults: a systematic review and meta-analysis. J Am Geriatr Soc 2004 Jul;52(7):1121-1129. [doi: 10.1111/j.1532-5415.2004.52310.x] [Medline: $\underline{15209650]}$

34. Stubbs B, Binnekade T, Eggermont L, Sepehry AA, Patchay S, Schofield P. Pain and the risk for falls in community-dwelling older adults: systematic review and meta-analysis. Arch Phys Med Rehabil 2014 Jan;95(1):175-187.e9. [doi: 10.1016/j.apmr.2013.08.241] [Medline: 24036161]

35. Dhital A, Pey T, Stanford MR. Visual loss and falls: a review. Eye (Lond) 2010 Sep;24(9):1437-1446. [doi: 10.1038/eye.2010.60] [Medline: 20448666]

36. Gruber T. Toward principles for the design of ontologies used for knowledge sharing. Int J Hum Comput Stud 1995;43(5-6):907-928.

37. Ferreira JD, Paolotti D, Couto FM, Silva MJ. On the usefulness of ontologies in epidemiology research and practice. J Epidemiol Community Health 2013 May;67(5):385-388 [FREE Full text] [doi: 10.1136/jech-2012-201142] [Medline: 23155060]

38. FARSEEING Project. 2015. URL: http://farseeingresearch.eu/ [accessed 2015-01-28] [WebCite Cache ID 6Vutz6H17]

39. Rubenstein LZ, Josephson KR. Falls and their prevention in elderly people: what does the evidence show? Med Clin North Am 2006 Sep;90(5):807-824. [doi: 10.1016/j.mcna.2006.05.013] [Medline: 16962843]

40. Heckerman D, Breese JSJ. A New Look at Causal Independence. 1994. URL: http://research.microsoft.com/en-us/um/ people/heckerman/hb94uai.pdf [accessed 2015-02-05] [WebCite Cache ID 6W79qXpPB]

41. Good I. A Causal Calculus. Br J Philos Sci 1961;11(44):305-318.

42. Zhang J, Yu KF. What's the relative risk? A method of correcting the odds ratio in cohort studies of common outcomes. JAMA 1998 Nov 18;280(19):1690-1691. [Medline: 9832001]

43. Lloyd JW. Foundations of logic programming. Berlin: Springer-Verlag; 1987.

44. Sato T. A statistical learning method for logic programs with distribution semantics. : MIT Press; 1995 Presented at: Twelfth International Conference on Logic Programming; 1995; Tokyo p. 715-729. 
45. LIACC/Universidade do Porto, Coppe Sistemas/UFRJ. Yet Another Prolog URL: http://www.dcc.fc.up.pt/ vsc/Yap/ [accessed 2015-01-28] [WebCite Cache ID 6VuvVd5W0]

46. InCHIANTI Group. 2014. The InCHIANTI Study Internet URL: http://inchiantistudy.net/wp/ [accessed 2014-11-05] [WebCite Cache ID 6TrTbShVW]

47. Hosmer DW, Lemeshow S, Sturdivant RX. Applied Logistic Regression. USA: John Wiley \& Sons; 2013.

48. Steyerberg EW. Clinical prediction models a practical approach to development, validation, and updating. New York: Springer; 2008.

49. Cattelani L, Palumbo P, Palmerini L, Chesani F, Chiari L. FRAT-up Web Application. 2014. URL: http://ffrat. farseeingresearch.eu/ [accessed 2014-12-31] [WebCite Cache ID 6VEgTdvOO]

50. Wyatt JJC, Altman DGD. Commentary: Prognostic models: clinically useful or quickly forgotten? BMJ 1995 Dec 09;311(7019):1539-1541. [doi: 10.1136/bmj.311.7019.1539]

51. Galán-Mercant A, Cuesta-Vargas AI. Differences in Trunk Accelerometry Between Frail and Nonfrail Elderly Persons in Sit-to-Stand and Stand-to-Sit Transitions Based on a Mobile Inertial Sensor. JMIR Mhealth Uhealth 2013;1(2):e21 [FREE Full text] [doi: 10.2196/mhealth.2710] [Medline: 25098977]

52. van Schooten KS, Sloot LH, Bruijn SM, Kingma H, Meijer OG, Pijnappels M, et al. Sensitivity of trunk variability and stability measures to balance impairments induced by galvanic vestibular stimulation during gait. Gait Posture 2011 Apr;33(4):656-660 [FREE Full text] [doi: 10.1016/j.gaitpost.2011.02.017] [Medline: 21435878]

53. Mellone S, Tacconi C, Chiari L. Validity of a Smartphone-based instrumented Timed Up and Go. Gait Posture 2012 May;36(1):163-165. [doi: 10.1016/j.gaitpost.2012.02.006] [Medline: 22421189]

54. Shany T, Redmond SJ, Marschollek M, Lovell NH. Assessing fall risk using wearable sensors: a practical discussion. A review of the practicalities and challenges associated with the use of wearable sensors for quantification of fall risk in older people. Z Gerontol Geriatr 2012 Dec;45(8):694-706. [doi: 10.1007/s00391-012-0407-2] [Medline: 23184295]

55. Mellone S, Tacconi C, Schwickert L, Klenk J, Becker C, Chiari L. Smartphone-based solutions for fall detection and prevention: the FARSEEING approach. Z Gerontol Geriatr 2012 Dec;45(8):722-727. [doi: 10.1007/s00391-012-0404-5] [Medline: 23184298]
Abbreviations
AUC: area under the ROC curve
FRAT-up: Fall-Risk Assessment Tool
LPAD: Logic Programming with Annotated Disjunctions
OR: odds ratio
POMA: Performance Oriented Mobility Assessment
ROC: receiver operating characteristic
TUG: Timed Up and Go Test

Edited by G Eysenbach; submitted 21.11.14; peer-reviewed by K van Schooten, S Deandrea; comments to author 11.12.14; revised
version received 08.01.15; accepted 10.01.15; published 18.02.15
Please cite as:
Cattelani L, Palumbo P, Palmerini L, Bandinelli S, Becker C, Chesani F, Chiari L
FRAT-up, a Web-based Fall-Risk Assessment Tool for Elderly People Living in the Community
JMed Internet Res $2015 ; 17(2): e 41$
URL: $\underline{\text { http://www.jmir.org/2015/2/e41/ }}$
doi: $\underline{10.2196 / j m i r .4064}$
PMID: 25693419

CLuca Cattelani, Pierpaolo Palumbo, Luca Palmerini, Stefania Bandinelli, Clemens Becker, Federico Chesani, Lorenzo Chiari. Originally published in the Journal of Medical Internet Research (http://www.jmir.org), 18.02.2015. This is an open-access article distributed under the terms of the Creative Commons Attribution License (http://creativecommons.org/licenses/by/2.0/), which permits unrestricted use, distribution, and reproduction in any medium, provided the original work, first published in the Journal of Medical Internet Research, is properly cited. The complete bibliographic information, a link to the original publication on http://www.jmir.org/, as well as this copyright and license information must be included. 